\title{
Ab initio Molecular Dynamics Investigations of the Speciation and Reactivity of Deep Eutectic Electrolytes in Aluminum Batteries
}

\author{
Dr. David Carrasco-Busturia/ Dr. Steen Lysgaard, Dr. Piotr Jankowski, \\ Prof. Tejs Vegge, Dr. Arghya Bhowmik, and Prof. Juan María García-Lastra† \\ Department of Energy Conversion and Storage, Technical University of Denmark, 2800 Kgs. Lyngby, Denmark
}

\begin{abstract}
Deep eutectic solvents (DESs) have emerged as an alternative for conventional ionic liquids in aluminum batteries. Elucidating DESs composition is fundamental to understand aluminum electrodeposition in the battery anode. Despite numerous experimental efforts, the speciation of these DESs remains elusive. This work shows how ab initio molecular dynamics (AIMD) simulations can shed light on the molecular composition of DESs. For the particular example of $\mathrm{AlCl}_{3}$ :urea, one of the most popular DESs, we carried out a systematic AIMD study, showing how an excess of $\mathrm{AlCl}_{3}$ in the $\mathrm{AlCl}_{3}$ :urea mixture promotes the stability of ionic species vs neutral ones and also favors the reactivity in the system. These two facts explain the experimentally observed enhanced electrochemical activity in salt-rich DESs. We also observe the transfer of simple $\left[\mathrm{AlCl}_{x}(\text { urea })_{y}\right]$ clusters between different species in the liquid, giving rise to free $\left[\mathrm{AlCl}_{4}\right]^{-}$units. The small size of these $\left[\mathrm{AlCl}_{4}\right]^{-}$ units favors the transport of ionic species towards the anode, facilitating the electrodeposition of aluminum.
\end{abstract}

Keywords: Ab initio Molecular Dynamics, Aluminum Batteries, Chemical Reactions, Deep Eutectic Solvent, Species Identification

\section{INTRODUCTION}

Today's modern society is facing an increased energy demand that must be addressed avoiding the use of fossilfuels and its effect in global warming. 10 3] Even though a number of renewable energy sources like wind and solar are becoming cost competitive with fossil resources for, e.g. electricity production, they remain inherently intermittent. 415 Hence, the development of new inexpensive and scalable energy-storage technologies has become a necessity targeted to an efficient use of renewables. Over the last decades, lithium-ion batteries have become the energy-storage system of choice, from small and portable devices up to electric vehicles and short-tomedium term stationary storage. $[$ Despite having the lowest reduction potential $(-3.045 \mathrm{~V}$ with respect to the standard hydrogen electrode), the Li-ion technology relies on critical raw materials (CRM) like cobalt. ${ }^{7}$ Postlithium energy storage systems is an area of active research, where aluminum is a promising candidate: it is abundant in the Earth's crust, and its volumetric capacity is approximately four times higher than that of lithium. $\frac{8}{8}$

Room temperature ionic liquids (RTILs) have been extensively investigated for battery electrolyte applications, both for Li- and Al-ion batteries, due to its high ionic conductivity, low flammability, and both thermal and chemical stability. 9-11 However, conventional RTILs, based on 1-ethyl-3-methylimidazolium (EMIM), are extremely expensive. For aluminum batteries, a much cheaper alternative to EMIM-based RTILs are deep eutectic solvents (DESs), based on aluminum chloride salts mixed

\footnotetext{
* dcabu@dtu.dk

$\dagger$ jmgla@dtu.dk
}

with simple organic molecules (e.g., urea - represented as "ur" thereafter in this work - or acetamide). $\frac{12}{12}$ Apart from involving low cost precursors in their preparation, DESs are highly biodegradable compared to EMIMbased RTILs.13/14 As a drawback, viscosities in DESs are typically at least one order of magnitude larger than those in EMIM-based RTILs. 12

A notorious difference in the electrochemical processes in aluminum batteries between EMIM-based RTILs and DESs is the Al-electrodeposition process. In EMIMbased RTILs the $\mathrm{Al}$ is electrodeposited in the negative electrode through a process in which only anions take part:

$$
4\left[\mathrm{Al}_{2} \mathrm{Cl}_{7}\right]^{-}+3 \mathrm{e}^{-} \rightarrow 7\left[\mathrm{AlCl}_{4}\right]^{-}+\mathrm{Al}
$$

By contrast, in DESs electrolytes, apart from the anionic process described in Equation (1), an electrodeposition in which cations get involved is also likely to happen. 15 In the particular case of the DESs based in urea, the following reaction may also occur: 16

$$
2\left[\mathrm{AlCl}_{2}(\mathrm{ur})_{2}\right]^{+}+3 \mathrm{e}^{-} \rightarrow \mathrm{Al}+\left[\mathrm{AlCl}_{4}\right]^{-}+4 \mathrm{ur}
$$

Further development of DESs requires to gain knowledge on the relative weight of these two reactions paths in $\mathrm{Al}$ electrodeposition. Another critical point is to understand the origin of the high viscosity of DESs in order to design possible strategies to remedy it. Prior to tackling these complex questions, two more fundamental ones have to be solved, namely: i) What is the chemical composition of the DES? and ii) How are the relevant species in the $\mathrm{Al}$ electrodeposition transported within the DES? The speciation and chemical reaction mechanisms of aluminum based DES electrolytes is still not well understood 
and a matter of ongoing debate. ${ }^{16}$ For the particular example of the aluminum chloride-urea DES, it is not clear what the concentration of neutral species vs. ionic ones is in the mixture nor what the relative amount of aluminum monomer ions (i.e., $\mathrm{AlCl}_{n}$ ) vs. aluminum dimer ions (i.e., $\mathrm{Al}_{2} \mathrm{Cl}_{m}$ ) or even longer $\mathrm{Al}$ chains is. $\mathrm{A}$ number of studies have shown that the electrodeposition of aluminum is enhanced when using DESs composed of $\mathrm{AlCl}_{3}$ and urea with the molar ratio $\mathrm{AlCl}_{3} /$ urea $>1$. $15|17| 18$ However, it is still not clear how $\mathrm{AlCl}_{3}$ / urea ratio affects the speciation of the DES. Regarding the second question, about the transport of the species participating in the $\mathrm{Al}$ electrodeposition, to the best of our knowledge no work has been reported in the literature.

The focus of this work is precisely to shed light on the speciation and transport mechanism in the DES formed by $\mathrm{AlCl}_{3}$ and urea by using state-of-the-art ab initio molecular dynamics (AIMD) simulations. Due to the complexity of the system we had to develop a specific computational framework to identify chemical species in the DES and track their reactivity. We find that the larger is the $\mathrm{AlCl}_{3}$ /urea ratio the more reactive is the ionic liquid and more ionic species relative to neutral ones are present. This aligns well with the experiments, where more electrochemical activity is observed for DES mixtures with an excess of aluminum salt. Regarding the transport of ions, we observe the ease to transfer aluminum monomer ions from one aluminum dimer to another, a process in which $\left[\mathrm{AlCl}_{4}\right]^{-}$is generated. This type of process facilitates the transport of ionic units towards the aluminum anode.

The paper is organized as follows. Section "Theoretical Method" describes the theoretical method, research approaches and strategy followed. The speciation and chemical reactions are investigated and discussed in section "Results and Discussion". and conclusions drawn in section "Conclusions" .

\section{THEORETICAL METHOD}

The reactivity and speciation of the ionic liquid has been analyzed for the 1:1 and 1.5:1 mole ratio mixtures of $\mathrm{AlCl}_{3}$ :urea. Ab initio molecular dynamics (AIMD) simulations within periodic boundary conditions were conducted with the VASP package ${ }^{[19}$ in the NVT ensemble, where the temperature is kept constant through the Nosé-Hoover thermostat. $\stackrel{20]}{ }$ In all the simulations the volume of the simulation box has been fixed so that it matches the experimental density at $333 \mathrm{~K}$. For the 1.5:1 mixture, the experimental density of $1.5921 \mathrm{~g} / \mathrm{cm}^{3} 21$ was used. No experimental density was found for the 1:1 mixture. An extrapolation of the densities reported in Ref. 21] for the 1.2:1, 1.3:1, 1.4:1 and 1.5:1 mixtures yields a density of 1.5398 for the $1: 1$ mixture $\mathrm{g} / \mathrm{cm}^{3}$, which is the one used in this work. The Verlet algorithm is used to integrate Newton's equations of motion with a time-step of 1 fs. The valence electrons are described by a plane-wave basis set with a $400 \mathrm{eV}$ energy cutoff. Five, six, one, four, three and seven valence electrons are included, respectively, for $\mathrm{N}, \mathrm{O}, \mathrm{H}, \mathrm{C}, \mathrm{Al}$ and $\mathrm{Cl}$ atoms. The core electrons are described by the projector augmented wave method, 22 combined with pseudopotentials. 23 The effects from exchange and correlation are modelled with the PBE functional 24 with the Langreth and Lundqvist van der Waals correlation as implemented in VASP. 25/26]. The break condition for the electronic self-consistent loop was set to $1 \cdot 10^{-4} \mathrm{eV}$ and the minimum number of electronic Self-Consistent Field (SCF) steps for each ionic step was set to 5. The electronic minimisation algorithm was set to RMM-DIIS. All calculations are performed using only the $\Gamma$ point in the reciprocal space.

Two different approaches have been followed in this work. The first approach (c.f. "reactants approach") is based on the direct AIMD simulation of $\mathrm{AlCl}_{3}$ and urea as a starting point. The second approach (c.f. "products approach") is based on the AIMD simulation of several proposed products (more information at the end of section "Reactants approach").

The complexity of DESs hinders the analysis of their composition and the tracking of the reactivity among the different species in these liquids. Similar to what Andersson et al. recently did for the analysis of highly concentrated electrolytes, ${ }^{27}$ here we had to develop a specific computational framework for this purpose. Sections "Procedure for identifying the species" and "Procedure for identifying the reactions" briefly describe the algorithms used to identify the molecules present in the DES and their reactivity, respectively.

\section{Reactants approach}

The 1.5:1 mixture is modelled as $18 \mathrm{AlCl}_{3}$ formula units and 12 molecules of urea in a cubic simulation box. In order to mimic the structure of a liquid, ideally all 21 molecules should initially be homogeneously distributed using all available space so that every molecule is given an equal opportunity to react with each other. Two different strategies were followed to guarantee this situation. The first strategy ensures a homogeneous arrangement of molecules through the PACKMOL program, 28 as implemented in the Amsterdam Density Functional (ADF) graphical user interface. 29 Several random seeds were initialized with this strategy. All these random seeds but one were discarded since chemically unstable chloroaluminate clusters were detected via the speciation procedure explained in section "Speciation". These species were predominant even after 100 ps time and are an artefact of starting at a relatively high temperature $(1000 \mathrm{~K})$. No such species appeared in one of the random seeds, which will be hereafter labelled as R1 and will be subject to analysis.

In view of these results, a second initialization strategy was designed. This strategy consists of randomly 
placing molecules of $\mathrm{Al}_{2} \mathrm{Cl}_{6}$ or urea in a grid of evenly distributed points (i.e., so that the minimum distance between any two points is as large as possible). Three random seeds were initialized with this strategy. Two random seeds were discarded because of the same reason described above. The third random seed, labelled as R2 in this paper, did not exhibit unstable chloroaluminate clusters and therefore was saved for analysis.

The 1:1 mixture is modelled as $14 \mathrm{AlCl}_{3}$ formula units and 14 molecules of urea in a cubic simulation box. Unfortunately, all three random seeds investigated yielded chemically unstable species. Hence, the 1:1 mixture was not considered for analysis within the reactants approach.

Both R1 and R2 reactants models were run for approximately $13 \mathrm{ps}$ at $1000 \mathrm{~K}$ to promote reactivity. Thereafter, the system is progressively cooled down to $300 \mathrm{~K}$ for 1 ps followed by a run at $300 \mathrm{~K}$. In total, this corresponds to an AIMD running history of $161 \mathrm{ps}$ (R1) and $151 \mathrm{ps}$ (R2).

In principle, one should expect that R1 and R2 should yield a similar composition, if they were run for long enough time. However, as it will be shown in section "Results and Discussion", models R1 and R2 result in significantly different compositions, even though both are apparently thermalized (i.e., no significant change in composition is observed in the last 20 ps of both runs). This points out that in order to have converged speciations, the simulations should have lasted and reached a time-scale well beyond nanoseconds, which is not feasible for this kind of systems using AIMD simulations. In view of this lack of convergence, and in addition to the aforementioned technical difficulties experienced in the construction of reactant models, a second approach was designed, i.e., the products approach, where the AIMD simulation starts from several products we hypothesize to be the most likely components of the ionic liquid based on experimental reports $\frac{15[18 \mid 30}{33}$ and chemical intuition. This strategy, presented below, aims to elucidate which of the hypothesized compositions is more stable.

\section{Products approach}

A number of Raman experiments have detected $\left[\mathrm{Al}_{2} \mathrm{Cl}_{7}\right]^{-}$and $\left[\mathrm{AlCl}_{4}\right]^{-}$, in the 1.5:1 mixture electrolyte. 15118 Some studies rationalize the occurrence of these chloroaluminate anions to participate in the intercalation/deintercalation in a graphite layered cathode. $30 \mid 31$ Others relate these to possible reactions taking place in a sulfur cathode during charging/discharging cycles. $\frac{32}{22}$ On the other hand, as we mentioned in the introduction, cationic species like $\left[\mathrm{AlCl}_{2}(\mathrm{ur})_{2}\right]^{+}$have been shown to participate in the electrodeposition of aluminum in the anode. $\frac{33}{33}$

The following reactions that we will present thereafter involve one or several of the above species found experimentally.

Three reactions have been considered for the 1.5:1 mix-

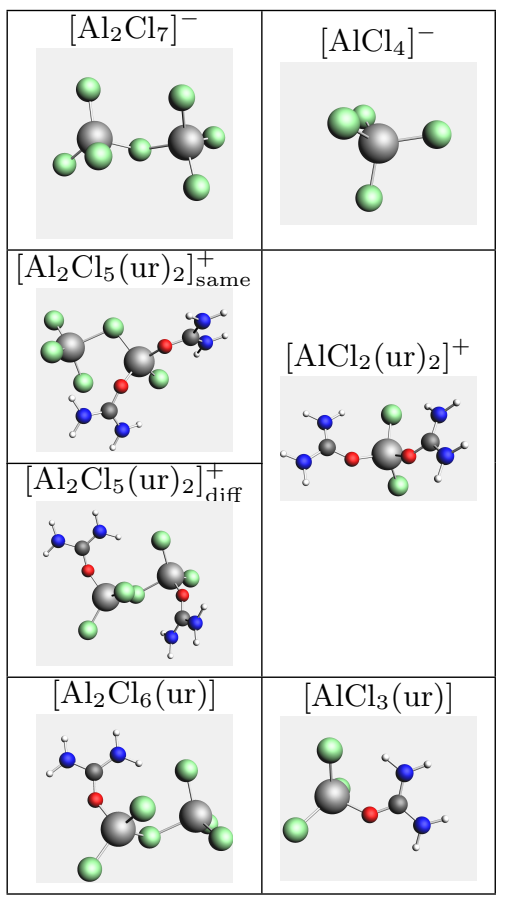

Figure 1. Species formulated throughout Equations (3)-(7) for the products approach.

ture:

$$
\begin{aligned}
& 3 \mathrm{AlCl}_{3}+2 \text { ur } \rightarrow\left[\mathrm{Al}_{2} \mathrm{Cl}_{7}\right]^{-}+\left[\mathrm{AlCl}_{2}(\mathrm{ur})_{2}\right]^{+} \\
& 3 \mathrm{AlCl}_{3}+2 \mathrm{ur} \rightarrow\left[\mathrm{AlCl}_{4}\right]^{-}+\left[\mathrm{Al}_{2} \mathrm{Cl}_{5}(\mathrm{ur})_{2}\right]^{+} \\
& 3 \mathrm{AlCl}_{3}+2 \text { ur } \rightarrow\left[\mathrm{Al}_{2} \mathrm{Cl}_{6}(\mathrm{ur})\right]+\left[\mathrm{AlCl}_{3}(\mathrm{ur})\right]
\end{aligned}
$$

Similarly, two reactions have been considered for the 1:1 mixture:

$$
\begin{aligned}
& 2 \mathrm{AlCl}_{3}+2 \mathrm{ur} \rightarrow\left[\mathrm{AlCl}_{3}(\mathrm{ur})\right]+\left[\mathrm{AlCl}_{3}(\mathrm{ur})\right] \\
& 2 \mathrm{AlCl}_{3}+2 \mathrm{ur} \rightarrow\left[\mathrm{AlCl}_{4}\right]^{-}+\left[\mathrm{AlCl}_{2}(\mathrm{ur})_{2}\right]^{+}
\end{aligned}
$$

The products in Equations (3)-(7) are presented in Figure 1 and were presented in a previous work we conducted. 16

The motivation for this approach is to model a 1.5:1 mixture formed by each of the two ionic pairs shown in the right hand side of Equations (3) and (4) on one hand, and the two neutral products from Equation (5) on the other hand. Similarly, a 1:1 mixture is modelled by the two neutral species shown in the right hand side of Equation (6) and the two ionic pair of products shown in Equation (7).

In the case of the products approach, since the fragments are ionic, initializing the simulation with a random distribution of molecules in the box (similar to that of the reactants approach) leads to enormous Coulombic 
repulsions. Thus, a special procedure for placing cationic and anionic fragments had to be designed so that the Coulombic attraction and repulsion forces were correctly balanced. A balanced spatial distribution of charges can be achieved by respectively placing these cationic and anionic fragments in the cationic and anionic positions of an ordered crystalline structure, e.g. $\mathrm{NaCl}$ type.

A special procedure was followed to place the ionic and neutral fragments into the rocksalt structure. Because of these fragments are large, a conventional substitution of these in the lattice positions of rocksalt structures typically yields to a situation where atoms are close in space and yet again large forces arise. Thus, all fragments need to be oriented in such a way that the interactions between neighbour atoms are reduced to a minimum. This has been achieved following a pairwise approach: given two fragments 1 and 2, the three Euler angles of the first fragment are rotated a certain angle so that the distance between all atoms of fragment 1 and all atoms of fragment 2 is minimized. In order to simplify the problem to computing the distance between atoms of two fragments only, we have worked over the rhombohedral primitive cell of $\mathrm{NaCl}$ (i.e., with $a=b=c$ and $\alpha=\beta=\gamma=60^{\circ}$ ). Once the fragments have been rotated to minimize interactions, a volume and shape constrained optimisation is conducted $(\mathrm{ISIF}=2$ setting in VASP) using a $2 \times 2 \times 2$ Monkhorst-Pack $k$-point mesh. 34 The resulting structure is then expanded to a $2 \times 2 \times 2$ supercell over which the AIMD simulations will be performed.

A similar approach has been followed taking as a model the $\mathrm{CsCl}$ lattice for all 1.5:1 and 1:1 products models. By the time 30 ps were reached, it was observed that in vast majority of the cases the energy was higher than the structures coming from the $\mathrm{NaCl}$ template. Because of limited computational resources, it was decided to reach the 120 ps goal only for those NaCl-based structures.

As it can be seen in Table 1, four models (P1, P2a, P2b, P3) for the 1.5:1 mixture and two models (P4, P5) for the 1:1 mixture are formulated with this approach, where 8 cationic and 8 anionic fragments form part of the starting AIMD structure.

Similar to the reactants approach, each of these models are heated at $1000 \mathrm{~K}$, but only for approximately 1.5 ps, since we consider the system is already starting from some sensible candidate products, and no extensive heating is required to promote reactivity as was the case with models $\mathrm{R} 1$ and $\mathrm{R} 2$ that start from the reactants $\mathrm{AlCl}_{3}$ and urea. A cool down to $300 \mathrm{~K}$ is conducted in $1 \mathrm{ps}$, followed by a run at $300 \mathrm{~K}$. In total, this corresponds to an AIMD running history of approximately 120 ps.

While only 168 atoms formed part of both mixtures in the reactants approach, the products approach consists of a total number of 224 and 192 atoms for the 1.5:1 and 1:1 mixtures respectively. Hence, the subsequent AIMD simulations for the products are computationally more demanding, particularly for the 1.5:1 mixture.

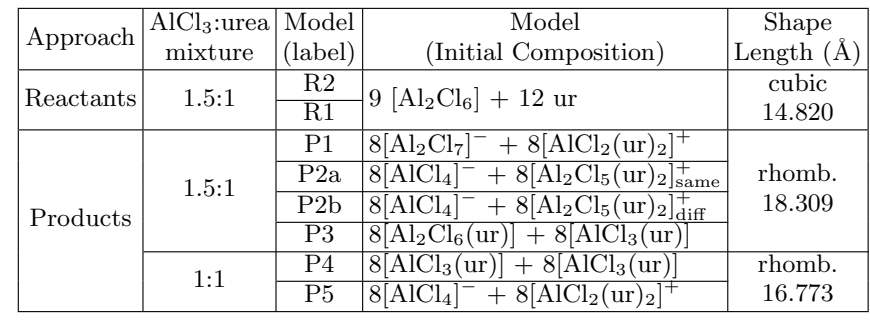

Table 1. Models studied in this work: reactants approach $\mathrm{R} 1$ and $\mathrm{R} 2$ for the 1.5:1 mixture and products approach $\mathrm{P} 1$, P2a, P2b, P3 (for the 1:1 mixture) and P4 and P5 (for the 1.5:1 mixture). The length and shapes (cubic and rhomb. for rhombohedral) of the simulation boxes are shown in the last column. The starting xyz configuration for each of these models are provided in the Supporting Information.

\section{Analysis tools}

As mentioned in the Introduction, the complexity of the $\mathrm{AlCl}_{3}$ :urea mixture led us to develop specific analysis tools to identify molecules (speciation - section "Speciation") and to discern between "open" and "closed" reactions (reactivity - section "Reactions"). The details of this framework are described below.

\section{Procedure for identifying the species}

As will be mentioned in section "Speciation", for each AIMD image, a connectivity matrix is constructed, where for any pair of $i$ and $j$ atom indices, the matrix elements read like:

$$
C_{i j}= \begin{cases}0 & \text { if } i \text { is not bonded to } j \\ 1 & \text { if } i \text { is bonded to } j\end{cases}
$$

Atoms $i$ and $j$ are bonded if the distance $d_{i, j}$ is less than the sum of their radii. The radius criteria assigned to each atom is the following: $\mathrm{Al}: 1 \AA, \mathrm{Cl}: 1.7 \AA, \mathrm{H}: 0.37$ $\AA, \mathrm{O}: 1 \AA, \mathrm{N}: 1 \AA, \mathrm{C}: 1 \AA$. Additionally, $\mathrm{Cl}$ atoms are only considered to be bonded to Al. The radii of the different species, which is a compromise between the ionic and the van der Waals radii of each element, was chosen such as the different species could be properly identified.

\section{Procedure for identifying the reactions}

As will be mentioned in section "Reactions", not only the speciation, but also the reactions taking place in the ionic liquid have been analyzed. By subtracting the matrix elements of the connectivity matrices involving two times $t_{1}$ and $t_{2}$, three cases can be found: 


$$
C_{i j}^{t_{2}}-C_{i j}^{t_{1}}=\left\{\begin{aligned}
-1 & \text { if bond } i-j \text { has been cleaved } \\
1 & \text { if bond } i-j \text { has been formed } \\
0 & \text { if bond } i-j \text { remains unchanged }
\end{aligned}\right.
$$

so that it is then possible to uniquely identify the indices of those atoms that form part of a reaction involving bond cleavage or formation.

The workflow of programs that both identify the species and track "open" and "closed" reaction mechanisms can be found in Ref. 35.

\section{RESULTS AND DISCUSSION}

\section{Speciation}

The identification of the species formed in the ionic liquid is described in this section. For each image of a given trajectory, a neighbour list and a subsequent connectivity matrix are constructed with the help of the Atomic Simulation Environment (ASE) ${ }^{36}$ software (more details in section "Procedure for identifying the species").

The trajectory is divided into consecutive blocks of 0.5 ps every 1 fs step. Both the average concentration of each species and the average energy are computed for each of these blocks as displayed in Figure 2. Results for the last $10 \mathrm{ps}$ are summarised in Table 2. The energy for each of the models has been normalized with respect to the energies of the solid phases of urea and $\mathrm{AlCl}_{3}$ as follows:

$$
\begin{aligned}
& E_{\text {Model }_{i}}=\frac{E\left({\text { AIMD } \left.\text { Model }_{i}\right)}_{N_{\text {ureas }}}\right.}{E\left(\mathrm{AlCl}_{3}(\mathrm{~s})\right)} \\
& -\left(n_{1} \frac{E\left(\mathrm{AlCl}_{3}(\mathrm{~s})\right)}{4 F . U .}+n_{2} \frac{E(\text { urea }(\mathrm{s}))}{2 F . U .}\right),
\end{aligned}
$$

where F.U. is the number of formula units in the solid phases, $n_{1}=1.5$ and $n_{2}=1$ for the $\mathrm{AlCl}_{3}$ :urea 1.5:1 mixture and $n_{1}=1$ and $n_{2}=1$ for the 1:1 mixture. $N_{\text {ureas }}$ is 16 and 12 for the products and reactants approach respectively. Positive energies indicate solid phases being more stable than the deep eutectic liquid.

Among the models constructed from the products approach, P2a is the most stable ionic liquid. The initial composition of $50 \%\left[\mathrm{AlCl}_{4}\right]^{-}, 50 \%\left[\mathrm{Al}_{2} \mathrm{Cl}_{5}(\mathrm{ur})_{2}\right]_{\text {same }}^{+}$decreases to $\approx 18 \%$ each, while $\left[\mathrm{AlCl}_{2}(\mathrm{ur})_{2}\right]^{+}$and $\left[\mathrm{Al}_{2} \mathrm{Cl}_{7}\right]^{-}$ composition rises to $31.3 \%$ each. The occurrence of these last two species can only be explained because $\left[\mathrm{Al}_{2} \mathrm{Cl}_{5}(\mathrm{ur})_{2}\right]_{\text {same }}^{+}$isomer allows to cleave in such a way that generates free $\left[\mathrm{AlCl}_{3}\right]$ and $\left[\mathrm{AlCl}_{2}(\mathrm{ur})_{2}\right]^{+}$(Figure 1). The reaction yield is far from $100 \%$ since $18.3 \%$ of $\left[\mathrm{Al}_{2} \mathrm{Cl}_{5}(\mathrm{ur})_{2}\right]_{\text {same }}^{+}$remains unbroken. On the other hand, no $\left[\mathrm{AlCl}_{3}\right]$ is observed in $\mathrm{P} 2 \mathrm{a}$, which suggests that all $\left[\mathrm{AlCl}_{3}\right]$ generated from that cleavage reacts with $\left[\mathrm{AlCl}_{4}\right]^{-}$ to produce $\left[\mathrm{Al}_{2} \mathrm{Cl}_{7}\right]^{-}(31.3 \%)$. The remaining $\left[\mathrm{AlCl}_{4}\right]^{-}$ sums up to $18.4 \%$.
P2a and P2b initial composition only differ on the type of $\left[\mathrm{Al}_{2} \mathrm{Cl}_{5}(\mathrm{ur})_{2}\right]^{+}$isomer. The structure of diff isomer present in $\mathrm{P} 2 \mathrm{~b}$ does not allow for a cleavage that would generate $\left[\mathrm{AlCl}_{2}(\mathrm{ur})_{2}\right]^{+}$or $\left[\mathrm{Al}_{2} \mathrm{Cl}_{7}\right]^{-}$(Figure 1), hence the occurrence of these two species in $\mathrm{P} 2 \mathrm{~b}$ is null. Instead, the diff isomer cleaves into $\left[\mathrm{AlCl}_{3}(\mathrm{ur})\right](18.2 \%)$ and $\left[\mathrm{AlCl}_{2}(\mathrm{ur})\right]^{+}(5.1 \%)$. Similar to P2a, the yield of this cleavage is not $100 \%$, since $\left[\mathrm{Al}_{2} \mathrm{Cl}_{5}(\mathrm{ur})_{2}\right]_{\text {diff }}^{+}$composition remains $28.1 \%$. The occurrence of $12.6 \%$ of $\left[\mathrm{Al}_{2} \mathrm{Cl}_{6}\right.$ (ur)] can be explained by the reaction of $\left[\mathrm{AlCl}_{3}(\mathrm{ur})\right]$ previously generated in the cleavage with $\left[\mathrm{AlCl}_{3}\right]$.

Despite Model P1 being almost isoenergetic with P2b, it scarcely generates new species different from the initial components at the investigated time-scales, i.e., its composition remains $\left[\mathrm{AlCl}_{2}(\mathrm{ur})_{2}\right]^{+}(48 \%)$ and $\left[\mathrm{Al}_{2} \mathrm{Cl}_{7}\right]^{-}$ (48\%). These are the same species that appear abundantly in $\mathrm{P} 2 \mathrm{a}$, which points out the fact that these two species are very likely to be formed in the 1.5:1 mixture as we hypothesized in a previous work, 16 where we studied isolated solvated pairs. In that work we observed the pair $\left[\mathrm{AlCl}_{2}(\mathrm{ur})_{2}\right]^{+}+\left[\mathrm{Al}_{2} \mathrm{Cl}_{7}\right]^{-}$to be the most stable one,

\begin{tabular}{|c|c|c|c|c|c|c|c|c|}
\hline & \multicolumn{6}{|c|}{ 1.5:1 mixture } & \multicolumn{2}{|c|}{ 1:1 mixture } \\
\hline & $\mathrm{R} 1$ & $\mathrm{R} 2$ & P1 & P2a & $\mathrm{P} 2 \mathrm{~b}$ & P3 & $\mathrm{P} 4$ & P5 \\
\hline Energy (eV) & 0.762 & 0.778 & 0.738 & 0.712 & 0.738 & 0.731 & 0.311 & 0.338 \\
\hline Speciation (\%) & & & & & & & & \\
\hline$\left[\mathrm{AlCl}_{2}(\mathrm{ur})_{2}\right]^{+}$ & 8.4 & 22.2 & 48.0 & 31.3 & & & & 50.0 \\
\hline$\left[\mathrm{Al}_{2} \mathrm{Cl}_{7}\right]^{-}$ & & 7.7 & 48.1 & 31.3 & & & & \\
\hline$\left[\mathrm{AlCl}_{4}\right]^{-}$ & 33.1 & 23.4 & 1.1 & 18.4 & 33.7 & 7.0 & & 50.0 \\
\hline$\left[\mathrm{Al}_{2} \mathrm{Cl}_{5}(\mathrm{ur})_{2}\right]^{+}$ & 16.4 & & 1.2 & 18.3 & 28.1 & & & \\
\hline$\left[\mathrm{AlCl}_{3}(\mathrm{ur})\right]$ & 8.4 & 23.3 & & & 18.2 & 62.5 & 100.0 & \\
\hline$\left[\mathrm{Al}_{2} \mathrm{Cl}_{6}\right.$ (ur)] & 16.7 & & & & 12.6 & 16.0 & & \\
\hline$\left[\mathrm{AlCl}_{2}(\mathrm{ur})\right]^{+}$ & & 6.4 & & & 5.1 & & & \\
\hline$\left[\mathrm{Al}_{3} \mathrm{Cl}_{9}(\mathrm{ur})_{2}\right]$ & & & 1.6 & & 1.7 & & & \\
\hline$\left[\mathrm{Al}_{5} \mathrm{Cl}_{15}(\mathrm{ur})_{2}\right]$ & & & & & & 6.5 & & \\
\hline$\left[\mathrm{Al}_{5} \mathrm{Cl}_{14}(\mathrm{ur})_{3}\right]^{+}$ & & & & & & 4.4 & & \\
\hline$\left[\mathrm{Al}_{3} \mathrm{Cl}_{9}(\mathrm{ur})\right]$ & & 15.5 & & & & & & \\
\hline$\left[\mathrm{AlCl}(\mathrm{ur})_{3}\right]^{2+}$ & 8.4 & & & & & & & \\
\hline$\left[\mathrm{Al}_{3} \mathrm{Cl}_{10}\right]^{-}$ & 8.3 & & & & & & & \\
\hline
\end{tabular}
with the pair $\left[\mathrm{Al}_{2} \mathrm{Cl}_{5}(\mathrm{ur})_{2}\right]_{\text {same }}^{+}+\left[\mathrm{AlCl}_{4}\right]^{-}$less than 0.1 $\mathrm{eV}$ above in energy.

Table 2. Energy and composition (in percentage) found in the last 10 ps trajectory for each models investigated for the 1.5:1 and 1:1 $\mathrm{AlCl}_{3}$ :urea mixtures. The energy has been normalized with respect to the energies of the solid phases of urea and $\mathrm{AlCl}_{3}$ as shown in Equation 10 .

Model P3, initially composed of $\left[\mathrm{AlCl}_{3}(\mathrm{ur})\right]$ and $\left[\mathrm{Al}_{2} \mathrm{Cl}_{6}\right.$ (ur)], predominantly evolves towards breaking the latter dimer into $\left[\mathrm{AlCl}_{3}(\mathrm{ur})\right]$ monomers $(62.5 \%)$. Despite exhibiting some reactivity, it is noticeable that this model is still mainly composed by neutral species (there is only approximately $10 \%$ of ionic species) and that its energy is well above that of model P2a (the most stable one), which is exclusively composed by ionic species.

The models coming from the 1:1 mixture (P4 and P5), show two striking differences with respect to those from the 1.5:1 mixture: i) 1:1 mixtures hardly present reactivity, since the initial composition is maintained throughout the trajectory. (i.e., P4: $100 \%\left[\mathrm{AlCl}(\mathrm{ur})_{3}\right]$ and $\mathrm{P} 5$ : 

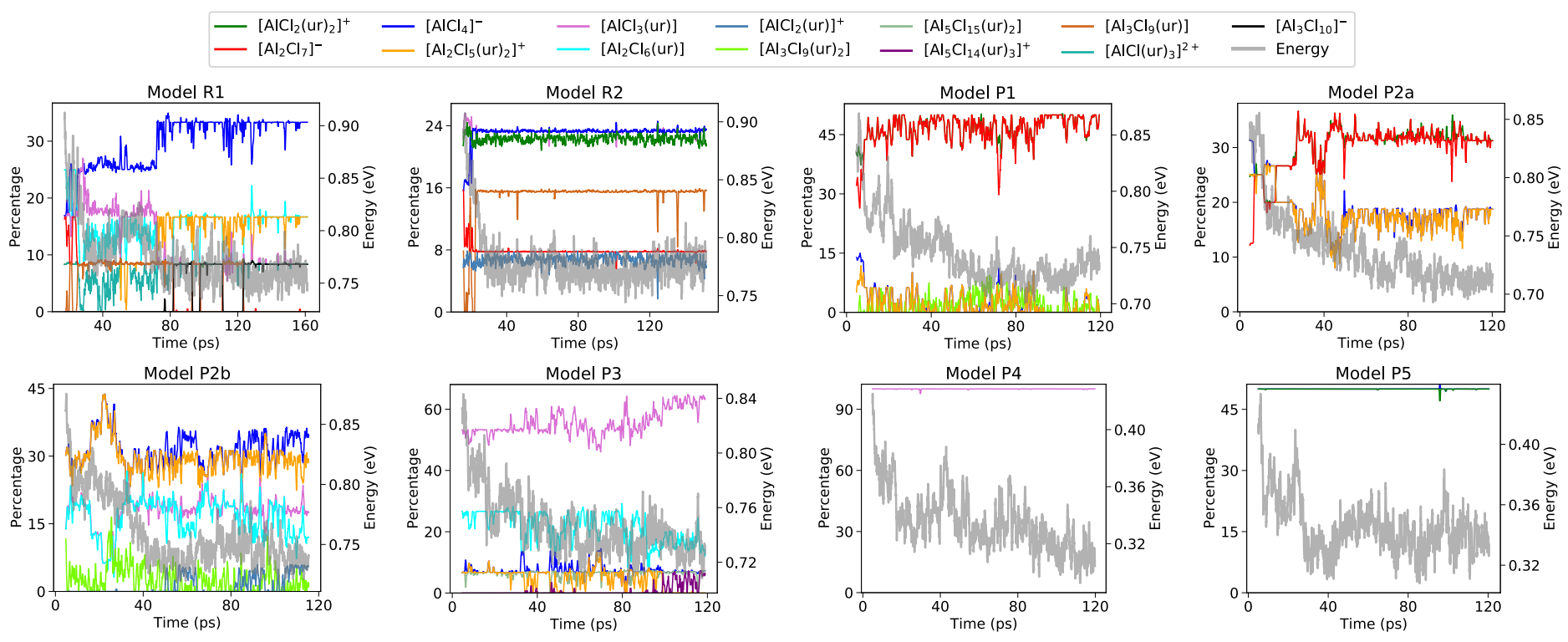

Figure 2. Energy and composition against time in picoseconds for each of the models studied in this work. The energy has been normalized with respect to the energies of the solid phases of urea and $\mathrm{AlCl}_{3}$ as shown in Equation (10).

$\left.50 \%\left[\mathrm{AlCl}_{2}(\mathrm{ur})_{2}\right]^{+}, 50 \%\left[\mathrm{AlCl}_{4}\right]^{-}\right)$. ii) The neutral model $(\mathrm{P} 4)$ is more stable than the ionic one (P5), in contrast to the 1.5:1 mixture. Thus, it is expected that the concentration of ionic species will be much higher in eutectic mixtures with an excess of salt, being this the explanation for the experimentally observed superior electrochemical activity in DESs with high salt/urea ratios.

Regarding the models constructed from the reactants approach, R1 is $0.015 \mathrm{eV}$ more stable than $\mathrm{R} 2$ and presents $\left[\mathrm{AlCl}_{4}\right]^{-}$as the most relevant species $(33.1 \%)$, followed by $\left[\mathrm{Al}_{2} \mathrm{Cl}_{6}\right.$ (ur) $](16.7 \%)$ and $\left[\mathrm{Al}_{2} \mathrm{Cl}_{5}(\mathrm{ur})_{2}\right]^{+}$ (16.4\%). On the other hand, three species are equally relevant in $\mathrm{R} 2$ model: $\left[\mathrm{AlCl}_{2}(\mathrm{ur})_{2}\right]^{+}(22.2 \%),\left[\mathrm{AlCl}_{4}\right]^{-}$ $(23.4 \%)$ and $\left[\mathrm{AlCl}_{3}(\mathrm{ur})\right](23.3 \%)$. It is remarkable that, despite having been run for a long period and having apparently reached equilibrium, models $\mathrm{R} 1$ and $\mathrm{R} 2$ yield significantly different compositions. In addition, the energies in R1 and R2 models are well above the energies in any of the product models. This points out the impossibility of obtaining any meaningful conclusion based on models initialized from the original components of the DES mixture if the time scale and size of the system are not well beyond what is affordable in AIMD simulations.

\section{Reactions}

A detailed study of the chemical reactions has been conducted for each of the models studied in this work. Because the indices of the atoms that participate in these reactions are tracked, it is possible to accurately evaluate if the indices involved in the cleavage/formation at a time $t_{1}$ participate in the reverse formation/cleavage reaction at any other given time $t_{2}$ (more details in section "Pro- cedure for identifying the reactions" If this scenario takes place, it has been named as a "closed reaction", which is no other than a chemical equilibrium. If otherwise, the situation has been classified as an "open reaction", where broken/formed indices never form/break back again.

"Closed reactions" are described in section "Closed reactions" of the Supporting Information. They typically involve $\mathrm{Al}-\mathrm{Cl}$ bond breaking and formation of long chains of $\left[\mathrm{Al}_{k} \mathrm{Cl}_{l}(\mathrm{ur})_{m}\right]$ with $k \geqslant 3$.

"Open reactions" have only been detected in models P2a, P2b and P3, and are summarized in Figure 3. Valuable information can be extracted from the analysis of these events. In model $\mathrm{P} 2 \mathrm{a}$, the charged species $\left[\mathrm{Al}_{2} \mathrm{Cl}_{7}\right]^{-}$ releases one neutral $\left[\mathrm{AlCl}_{3}\right]$ unit to be captured by the charged species $\left[\mathrm{AlCl}_{2}(\mathrm{ur})_{2}\right]^{+}$to form $\left[\mathrm{Al}_{2} \mathrm{Cl}_{5}(\mathrm{ur})_{2}\right]_{\text {same }}^{+}$. This reaction occurs through a $\left[\mathrm{Al}_{3} \mathrm{Cl}_{9}(\mathrm{ur})_{2}\right]_{\text {same }}$ transition state that lives $167 \mathrm{fs}$. This type of open reaction can be classified as the transfer of one neutral species $\left(\left[\mathrm{AlCl}_{3}\right]\right)$ released from one charged species $\left(\left[\mathrm{Al}_{2} \mathrm{Cl}_{7}\right]^{-}\right)$, to be captured by another charged species $\left(\left[\mathrm{AlCl}_{2}(\mathrm{ur})_{2}\right]^{+}\right)$. The opposite process can also occur, and is indeed the mechanism seen in model P2b (Figure 3): the transfer of one charged species $\left(\left[\mathrm{AlCl}_{2}(\mathrm{ur})\right]^{+}\right)$ released from one neutral species $\left(\left[\mathrm{Al}_{2} \mathrm{Cl}_{6}(\mathrm{ur})\right]\right)$, to be captured by another neutral species $\left(\left[\mathrm{AlCl}_{3}(\mathrm{ur})\right]\right)$. Unlike the previous case, the unit released is not neutral but charged $\left(\left[\mathrm{AlCl}_{2}(\mathrm{ur})\right]^{+}\right)$, and has a lifetime of $12 \mathrm{fs}$.

Both mechanisms described so far show the relatively ease for this ionic liquid to generate low diffusivity $\left[\mathrm{AlCl}_{4}\right]^{-}$ions, which opens up the possibility for a faster anionic transport across the electrolyte, since the diffusivity of smaller $\left[\mathrm{AlCl}_{4}\right]^{-}$is expected to be much faster than that of $\left[\mathrm{Al}_{2} \mathrm{Cl}_{7}\right]^{-}$. Such diffusion of negatively charged chloroaluminate ions is key to promote the elec- 

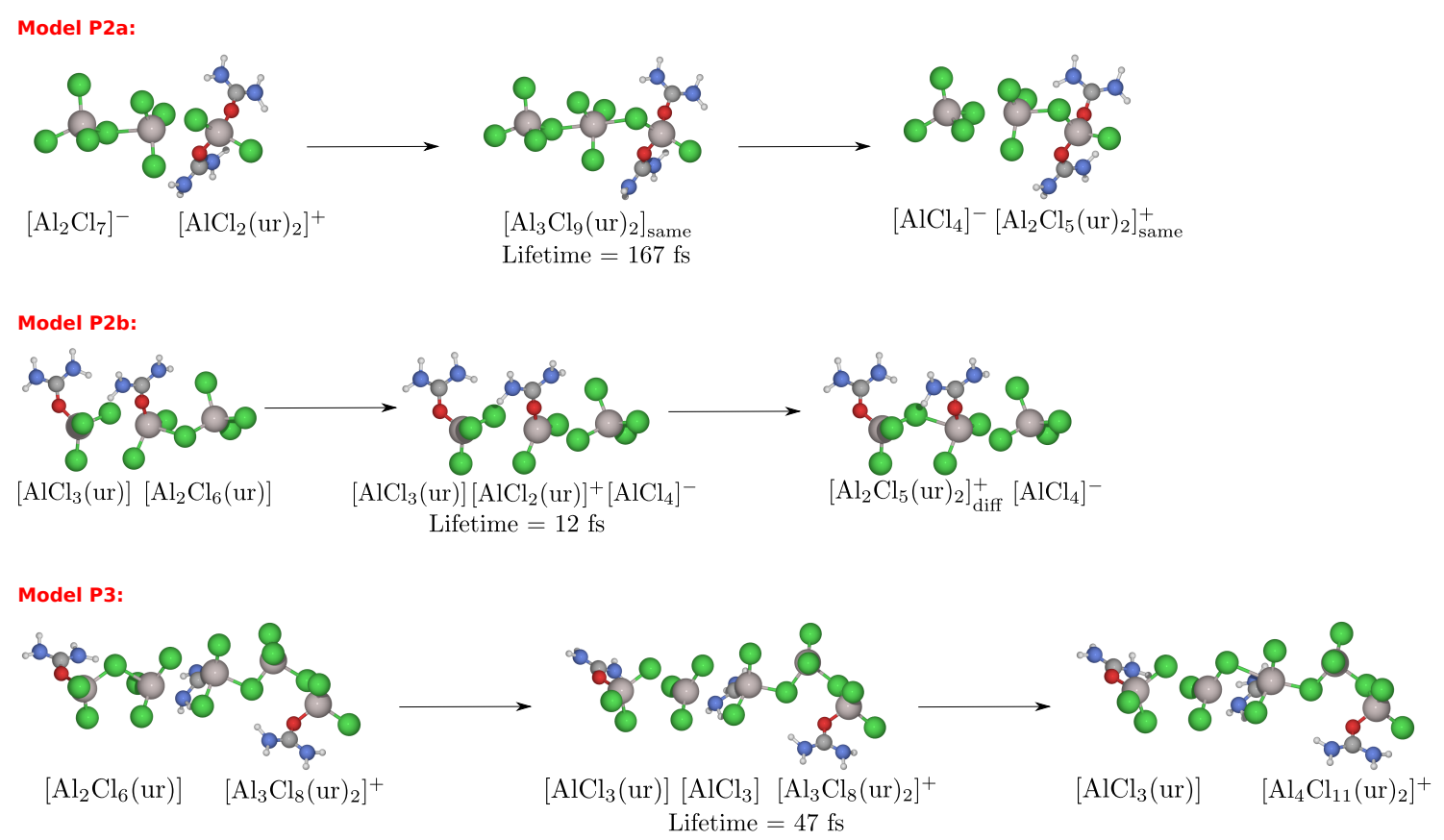

Figure 3. Open reactions observed in the last 10 ps trajectory for models P2a, P2b and P3. No open reactions have been reported for the rest of the models. Three videos and trajectory files showing the mechanism of these three reactions are included in the Supporting Information.

trochemistry at the electrode interface.

A hybrid mechanism, combination of the above two, has also been detected in model P3, where the transfer of neutral $\left(\left[\mathrm{AlCl}_{3}\right]\right)$ takes place between neutral $\left(\left[\mathrm{Al}_{2} \mathrm{Cl}_{6}\right.\right.$ (ur) $\left.]\right)$ and charged $\left(\left[\mathrm{Al}_{3} \mathrm{Cl}_{8}(\mathrm{ur})_{2}\right]^{+}\right)$units.

\section{CONCLUSIONS}

We performed AIMD simulations to shed light on the composition of the $\mathrm{AlCl}_{3}$ :urea DES. Due to the complexity of the liquid we had to develop a computational machinery for the identification of species and tracking of chemical reactions, which could be useful to investigate similar DESs. In a first attempt, we set up the AIMD model starting from the reactants in the DES, $\mathrm{AlCl}_{3}$ and urea, heating the system to promote reactivity. This method resulted in being impractical, showing unreliable speciation, or giving rise to high energy configurations, even after running for more than 100 ps. In a more pragmatic approach, we carried out AIMD simulations starting from different sets of products that have been experimentally observed in this DES and compared their relative energies. This methodology allowed us to extract relevant conclusions about the $\mathrm{AlCl}_{3}$ :urea DES speciation and reactivity, namely:

- The stability of ionic pairs vs. neutral species and the reactivity of the DES increase notably upon increasing the amount of aluminum salt vs. urea in the deep eutectic mixture. This explains the experimentally observed enhanced electrochemical activity in DESs with a high $\mathrm{AlCl}_{3}$-to-urea ratio.

- We observed the transfer of simple $\left[\mathrm{AlCl}_{x}(\text { urea })_{y}\right]$ clusters, both neutral and charged, between different species in the liquid, giving rise to free $\left[\mathrm{AlCl}_{4}\right]^{-}$ units. We hypothesized this as a plausible mechanism to enhance the transport of electrochemically relevant species, such as $\left[\mathrm{AlCl}_{4}\right]^{-}$, from the bulk of the electrolyte towards the anode.

- For the 1.5:1 $\mathrm{AlCl}_{3}$ :urea DES, the most relevant from the experimental point of view, we found a purely ionic model formed by approximately $2 / 3$ of $\left[\mathrm{AlCl}_{2}(\mathrm{ur})_{2}\right]^{+}+\left[\mathrm{Al}_{2} \mathrm{Cl}_{7}\right]^{-}$pairs and $1 / 3$ of $\left[\mathrm{AlCl}_{4}\right]^{-}+\left[\mathrm{Al}_{2} \mathrm{Cl}_{5}(\mathrm{ur})_{2}\right]^{+}$pairs to be the most stable one.

The most stable composition points out that in principle, both the anionic and cationic paths for $\mathrm{Al}$ electrodeposition sketched in Equations (1) and (2) could be possible in the 1.5:1 $\mathrm{AlCl}_{3}$ :urea DES. Elucidating which of the two routes is more favorable would require AIMD simulations explicitly including the aluminum anode and spanning much longer time and length scales than those presented in this work. The cost of such models would be prohibitive using conventional AIMD calculations. We envisage machine learning potentials, trained on data like the one presented here, as a viable route to carry out such simulations. Further work along this line is now underway. 


\section{ACKNOWLEDGMENTS}

This work was funded by the European Union H2020 SALBAGE and AMAPOLA projects (grant agreements No. 766581 and 951902 respectively).

[1] D. Larcher, J.-M. Tarascon, Nat. Chem. 2014, 7, 19-29.

[2] H. Nazir, M. Batool, F. J. B. Osorio, M. Isaza-Ruiz, $\mathrm{X} . \mathrm{Xu}, \mathrm{K}$. Vignarooban, P. Phelan, Inamuddin, A. M. Kannan, Int. J. Heat Mass Transfer 2019, 129, 491 523.

[3] S. Yun, Y. Zhang, Q. Xu, J. Liu, Y. Qin, Nano Energy 2019, 60, $600-619$.

[4] Z. Yang, J. Zhang, M. C. W. Kintner-Meyer, X. Lu, D. Choi, J. P. Lemmon, J. Liu, Chem. Rev. 2011, 111, 3577-3613.

[5] X. Zhou, Q. Liu, C. Jiang, B. Ji, X. Ji, Y. Tang, H.-M. Cheng, Angew. Chem. Int. Ed. Engl. 2020, 59, 38023832.

[6] Y. Hu, D. Sun, B. Luo, L. Wang, Energy Technol. 2019 , 7, 86-106.

[7] J. Wang, Y. Hu, Y. Li, X. Gao, X. Wu, Z. Wen, J. Power Sources 2020, 453, 227843.

[8] H. Yang, H. Li, J. Li, Z. Sun, K. He, H.-M. Cheng, F. Li, Angew. Chem. Int. Ed. Engl. 2019, 58, 11978-11996.

[9] M. Armand, F. Endres, D. R. MacFarlane, H. Ohno, B. Scrosati, Nat. Mater. 2009, 8, 621-629.

[10] G. A. Elia, K. Marquardt, K. Hoeppner, S. Fantini, R. Lin, E. Knipping, W. Peters, J.-F. Drillet, S. Passerini, R. Hahn, Adv. Mater. 2016, 28, 7564-7579.

[11] S. Das, J. Højberg, K. B. Knudsen, R. Younesi, P. Johansson, P. Norby, T. Vegge, J. Phys. Chem. C 2015, 119, 18084-18090.

[12] A. P. Abbott, G. Capper, D. L. Davies, K. J. McKenzie, S. U. Obi, J. Chem. Eng. Data 2006, 51, 1280-1282.

[13] D. Lloyd, T. Vainikka, L. Murtomäki, K. Kontturi, E. Ahlberg, Electrochim. Acta 2011, 56, $4942-4948$.

[14] M. Landa-Castro, J. Aldana-González, M. Montes de Oca-Yemha, M. Romero-Romo, E. Arce-Estrada, M. Palomar-Pardavé, J. Alloys Compd. 2020, 830, 154650

[15] M. Angell, C.-J. Pan, Y. Rong, C. Yuan, M.-C. Lin, B.J. Hwang, H. Dai, Proc. Natl. Acad. Sci. U. S. A. 2017, $114,834-839$.

[16] Á. Miguel, R. P. Fornari, N. García, A. Bhowmik, D. Carrasco-Busturia, J. M. García-Lastra, P. Tiemblo, ChemSusChem 2020, 13, 5523-5530.

[17] K. S. Ryder, C. Zaleski, I. Efimov, T. Purnell, ECS Meeting Abstracts, 2019.
[18] M. Angell, G. Zhu, M.-C. Lin, Y. Rong, H. Dai, Adv. Funct. Mater. 2020, 30, 1901928.

[19] G. Kresse, J. Furthmüller, Phys. Rev. B 1996, 54, 11169-11186.

[20] S. Nosé, J. Chem. Phys. 1984, 81, 511-519.

[21] C. Liu, W. Chen, Z. Wu, B. Gao, X. Hu, Z. Shi, Z. Wang, J. Mol. Liq. 2017, 247, $57-63$.

[22] P. E. Blöchl, Phys. Rev. B 1994, 50, 17953-17979.

[23] G. Kresse, D. Joubert, Phys. Rev. B 1999, 59, 17581775 .

[24] J. P. Perdew, K. Burke, M. Ernzerhof, Phys. Rev. Lett. 1996, 77, 3865-3868.

[25] J. Klimeš, D. R. Bowler, A. Michaelides, Phys. Rev. B 2011, 83, 195131.

[26] J. Klimeš, D. R. Bowler, A. Michaelides, J. Phys.: Condens. Matter 2009, 22, 022201.

[27] R. Andersson, F. Årén, A. A. Franco, P. Johansson, J. Electrochem. Soc. 2020, 167, 140537.

[28] J. I. Rodríguez, A. M. Köster, P. W. Ayers, A. SantosValle, A. Vela, G. Merino, J. Comput. Chem. 2009, 30, 1082-1092.

[29] G. te Velde, F. M. Bickelhaupt, E. J. Baerends, C. Fonseca Guerra, S. J. A. van Gisbergen, J. G. Snijders, T. Ziegler, J. Comput. Chem. 2001, 22, 931-967.

[30] C. Wang, J. Li, H. Jiao, J. Tu, S. Jiao, RSC Adv. 2017, 7, 32288-32293.

[31] H. Jiao, C. Wang, J. Tu, D. Tian, S. Jiao, Chem. Commun. 2017, 53, 2331-2334.

[32] Y. Bian, Y. Li, Z. Yu, H. Chen, K. Du, C. Qiu, G. Zhang, Z. Lv, M.-C. Lin, ChemElectroChem 2018, 5, 3607-3611.

[33] A. P. Abbott, R. C. Harris, Y.-T. Hsieh, K. S. Ryder, I.-W. Sun, Phys. Chem. Chem. Phys. 2014, 16, 1467514681.

[34] J. D. Pack, H. J. Monkhorst, Phys. Rev. B 1977, 16, 1748-1749.

[35] Workflow of programs that both identify the species and track open and closed reaction mechanisms, https : / / github . com / DavidCdeB / Chemical-Speciation-and-Reaction-Mechanisms-Identification

[36] A. H. L. et. al, J. Phys.: Condens. Matter. 2017, 29, 273002. See Supporting Information.

INSTITUTE AND/OR RESEARCHER TWITTER USERNAMES:

- @DavidCdeB

- @StLysgaard

- @piotr_portos 
- @ArghyaBhowmik5

- @TVegge

- @JuanMaraGarcaL1

- @AMAPOLA_project

- @SALBAGE_Project

- @DTUEnergy

- @DTUtweet 